\title{
Stylized facts from a threshold-based heterogeneous agent model
}

\author{
R. Cross \\ Department of Economics, University of Strathclyde, \\ Sir William Duncan Building, 130 Rottenrow Glasgow G4 OGE, Scotland, UK \\ M. Grinfeld \\ Department of Mathematics, University of Strathclyde, \\ Livingstone Tower, 26 Richmond Street, Glasgow G1 1XH, Scotland, UK \\ H. Lamba \\ Department of Mathematical Sciences, George Mason University, 4400 University Drive, Fairfax, VA 22030 USA \\ T. Seaman \\ School of Computational Sciences, George Mason University, 4400 University Drive, Fairfax, VA 22030 USA
}

(Dated: August 1, 2006)

\begin{abstract}
A class of heterogeneous agent models is investigated where investors switch trading position whenever their motivation to do so exceeds some critical threshold. These motivations can be psychological in nature or reflect behaviour suggested by the efficient market hypothesis (EMH). By introducing different propensities into a baseline model that displays EMH behaviour, one can attempt to isolate their effects upon the market dynamics.

The simulation results indicate that the introduction of a herding propensity results in excess kurtosis and power-law decay consistent with those observed in actual return distributions, but not in significant long-term volatility correlations. Possible alternatives for introducing such long-term volatility correlations are then identified and discussed.
\end{abstract}

PACS numbers: 89.65.Gh 89.75.Da

\section{INTRODUCTION}

It is hard to overestimate the impact that the concept of efficient markets has had on economic and political thinking. The underlying efficient market hypothesis (EMH) [1] has enormous philosophical and mathematical appeal but is perhaps best thought of as a Platonic ideal. The strong form of the hypothesis is that investors have access to all relevant information, and that this is fully reflected by the current market price. The random arrival of new (independent and identically Gaussian-distributed) information causes traders' expectations to change. This is then translated into a Brownian motion in, and a Gaussian distribution of, (log) price returns. There are variations upon the above reasoning, for example, invoking arbitrageurs or 'informed' investors who quickly exploit any inefficiencies due to 'noise' traders or 'uninformed' investors but the pricing outcome is the same. One of the refutable implications of the EMH is the Gaussian distribution of returns. Actual distributions however are sufficiently non-Gaussian so as to require better explanations and mathematical models than provided by the EMH [2, 3].

Two types of assumptions underlie the EMH. Firstly, there are assumptions about the nature of the information entering the system (for example, its stationarity and lack of correlations), the dissemination of this data amongst the market participants, and their ability to evaluate and react to it. Given the enormous increase in information processing speeds, and the rise of instantaneous mass global communication, it is not implausible to suppose that some EMH violations of this type have become less important over recent decades.

The second set of assumptions concerns the rationality and motivations of the agents themselves, be they individuals or financial institutions. As regards individuals, recent work by psychologists and experimental economists has suggested that deviations from expected utility maximisation are widespread, even when 'smart' people are playing 'simple' games. Furthermore, there are structural and institutional features that can undermine the EMH. Examples include compensation/evaluation/bonus criteria, tax laws, accounting rules, conflicts of interest within a financial organization and moral hazards problems.

With so many plausible EMH violations (and the impossibility of performing controlled experiments with real markets), it is extremely difficult to draw conclusions regarding the chain of cause and effect from statistical analyses alone. However these analyses have identified a set of 'stylized facts' that appear to be prevalent across asset classes independent of trading rules, geography or culture. These include the lack of linear correlations in price returns over all but the shortest timescales, excess kurtosis (fat-tails) in the price return distribution, volatility clustering and heteroskedasticity. Some finer details have also been revealed, most notably the existence of power-law scalings and estimates of the exponents. 
The class of models presented here (see also [4, [5, 6]) is an attempt to provide a framework within which to study systematically the effects of various, simple, EMH violations. The hope is that the insights gained will result in both a greater theoretical understanding of the operation of markets and in better simulation tools for market practitioners. The modeling process we advocate is based upon the idea of thresholds. At each point in time, agents are comfortable with their current position (either long or short on the market). However, they are subject to one or more 'tensions' which cause a switch in position whenever the corresponding threshold is violated. The use of the word 'tension' does not necessarily imply that the response is emotional or psychological in nature (although it may be) — the agent may have buy/sell price triggers in place based upon analytical research, in which case the tension level merely reflects the distance from the current price to the closest threshold.

These models, together with a related approach that can be applied to Minority Games 7], have been introduced elsewhere [4, [5] and the reader is directed to them for further details. The main contributions of this paper are to more thoroughly consider the modeling of volatility clustering and examine the relative performances of the heterogeneous agents. The paper is organized as follows. In Section $\amalg$ we introduce a minimal, baseline, model in which the market price remains identical to a market operating under the EMH. By including additional tensions one can then observe the corresponding changes in the market statistics. This is performed in Section IIII where a herding propensity is included, resulting in fat-tails and excess kurtosis, but no long-term volatility correlations. In Section [V] we discuss different possibilities for generating volatility clustering in the form of slowly-decaying correlations. Finally, in Section $\square$ the relative performance of agents with differing herding propensities is investigated.

\section{A THRESHOLD MODEL WITH EMH PRICE RETURNS}

The system evolves in discrete timesteps of length $h$ (which will be chosen to correspond to one trading day for the simulations in this paper). There are $M$ agents, all of equal size, who can be either long or short in the market over the $n^{\text {th }}$ time interval. The market price at the end of the $n^{\text {th }}$ time interval is $p(n)$. For simplicity $p(0)=1$ and we assume that the system is drift-free so that, in reality, $p(n)$ corresponds to, say, the price corrected for the risk-free interest rate plus equity-risk premium or the expected rate of return. The position of the $i^{t h}$ investor over the $n^{\text {th }}$ time interval is represented by $s_{i}(n)= \pm 1$ ( +1 long, -1 short), and the sentiment of the market by the average of the states of all of the $M$ investors

$$
\sigma(n)=\frac{1}{M} \sum_{i=1}^{M} s_{i}(n) .
$$

The change in market sentiment from the previous time interval is defined by $\Delta \sigma(n)=\sigma(n)-\sigma(n-1)$.

Before defining the model we make the following important point. We are not attempting to simulate directly all of the market participants, just those whose trading strategies are most significant over the timescale of interest. Thus we start by hypothesizing the existence of some underlying EMH market and change as little as possible. In particular we shall assume that arbitrageurs and traders exist who act to interpret the incoming information stream and induce the corresponding price changes over timescales $\ll h$. Other market details, such as the way in which orders are placed and executed, remain unspecified but constant.

We shall also assume a simple linear relationship between changes in the sentiment $\Delta \sigma$ and the excess pricing pressure it induces. This leads us to the following geometric pricing formula

$$
p(n+1)=p(n) \exp (\sqrt{h} \eta(n)-h / 2+\kappa \Delta \sigma(n))
$$

where $\sqrt{h} \eta(n) \sim \mathcal{N}(0, h)$ represents the exogenous information stream. The parameter $\kappa$ reflects the relative effects on price of internally generated dynamics as opposed to the information. Finally, the term $-h / 2$ is the drift correction required by Itô calculus to ensure that, for $\kappa=0$, the price $p(t)$ is a martingale. It can be safely omitted from the model but we choose to include it here for completeness.

In order to close the model we must now specify how the states of the individual agents are determined, i.e. how the $i^{\text {th }}$ agent decides when to switch. This is achieved by introducing an 'inaction' pressure. Every time the agent switches position a pair of threshold prices on either side of the current price is generated. When the current market price crosses one of these threshold values the agent switches once again, a new pair of thresholds is generated and the process repeats (more generally, the thresholds can be updated continuously rather than only when the agent switches but this appears to make little difference to the behaviour of the model). An appealing feature of the inaction pressure is that it is capable of multiple interpretations - at the 'rational' end of the spectrum, the price interval defined by the thresholds corresponds to an investment strategy based upon the market analysis and future expectations of that agent. Other effects that can also be reproduced, are: the psychological factors behind the desire to cut losses or 
take profits; transaction costs and the resulting hysteresis effects; the irrational need for agents to do something or the (less ir)rational need to be seen to be doing something (in the case of active-fund managers, perhaps). Further details can be found in [4].

To define the model precisely, let $P_{i}$ be the price at which the $i^{\text {th }}$ investor last switched positions and let $H_{i}>0$ be a value, chosen randomly at each switching from the uniform distribution on the interval $\left[H_{\mathrm{L}}, H_{\mathrm{U}}\right]$. Then, as long as the current price $p(n)$ stays within the interval $\left[P_{i} /\left(1+H_{i}\right), P_{i}\left(1+H_{i}\right)\right]$, the investor maintains her position, but if the current price $p(n)$ leaves this interval, the investor switches. The choice of a uniform distribution is made purely on grounds of simplicity - the model appears to be extremely robust and, in the absence of other information, there is nothing to be gained by making the model more complicated than necessary.

The behaviour of the above model is reasonably straightforward. Provided that $M$ is sufficiently large $(M=100$ appears to be sufficient), and that the initial agent states are sufficiently mixed with $\sigma(0) \approx 0$, sentiment will remain close to 0 and the price remains close to its fundamental EMH value. This is because there is no coupling between agents and their switches in position cancel without affecting the sentiment [ 8 ]. Thus we have a model that is very close, both philosophically and in appearance, to that posited by the EMH - the price follows a geometric Brownian motion and, if one interprets the inaction pressure in the 'rational' way described above, trading is induced by the differing expectations of agents. We hesitate to describe the model as efficient since the volume of trading is determined solely by the interval $\left[H_{\mathrm{L}}, H_{\mathrm{U}}\right]$. This implies that excess trading may occur which is inefficient in the presence of transaction costs. However such excess trading is another well-documented feature of actual financial markets [9].

\section{INCORPORATING A HERDING PRESSURE}

There are other pressures affecting investors which, when included in the model, will not not necessarily cancel out, most likely due to some form of global coupling. The simplest, and arguably the single most important, example of such a pressure is the 'herding tendency' — while an individual/organization is holding a minority opinion/position they may feel an increasing pressure to conform that eventually becomes unbearable (unless enough of the agents with majority positions switch first), at which point they will switch to join the majority. Clearly different agents will have different tolerance levels that are, to some extent, a reflection of their personality or trading philosophy (such as 'momentum traders' and 'contrarian investors'). Although it is tempting to describe such herding behaviour as irrational, or 'boundedly-rational' in the sense of Simon [10, 11], this may not be a fair characterization in all cases. Some agents may lose their job/investment capital if they significantly underperformed the average market or benchmark return for even a few quarters in a row - such agents are exhibiting behaviour that is no more irrational than animals herding when surrounded by predators 12 .

We incorporate the herding tendency as follows. At time $n$, the herding pressure felt by agent $i$ is denoted by $c_{i}(n)$. This level is changed to $c_{i}(n+1)=c_{i}(n)+h|\sigma(n)|$ (i.e. is increased by an amount proportional to the length of the time interval and the severity of the inconsistency) whenever $s_{i}(n) \sigma(n)<0$. Otherwise, the agent's herding pressure remains unchanged and $c_{i}(n+1)=c_{i}(n)$. As soon as $c_{i}(n)$ exceeds her (constant) threshold $C_{i}$, the investor switches market position and $c_{i}$ is reset to zero. Additionally we suppose that whenever a switch occurs, both the inaction and herding pressures are set to zero (although the model appears to be very robust with respect to such changes in the interactions between the tensions $[4,[5])$.

We now choose some realistic parameters and present some numerical results. A daily variance in price returns of $0.6-0.7 \%$ suggests a value for $h$ of 0.00004 . The number of participants $M=100$ and it is worth noting that the model's characteristics are independent of $M$ - this is an important property not always shared by other heterogeneous agent models. The simulation is run for 10000 timesteps which corresponds to approximately 40 years of trading.

Once $h$ has been fixed, we suppose that the $C_{i}$ are chosen from the uniform distribution on [0.001,0.004], as this leads to herd-induced switching on the timescale of weeks and months for those agents in the minority. The price ranges for the inaction tension are chosen randomly after every switching from the uniform distribution on the interval $10 \%-30 \%$, i.e. $\left[H_{L}, H_{U}\right]=[0.1,0.3]$. Day-traders would of course have much smaller values but our choice of $h$ means that we cannot attempt to model directly changes occurring over such short timescales. Finally, simulations using the above parameters suggest that a value of $\kappa=0.2$ results in prices that are strongly correlated with the information stream but which differ significantly during periods of extreme market sentiment.

Figure \shows the output of a typical run. Figure \) plots the price $p(t)$ against the 'fundamental' price obtained by setting $\kappa=0$ (which decouples the price from the agent dynamics and generates a pure geometric Gaussian price stream). It should be noted that the agents typically switch every few weeks or months and that the vast majority of trades are due to the inaction thresholds being violated. However the sentiment $\sigma$, as can be seen in Figure 1b), changes more slowly and can remain bullish or bearish for several years. Figure 1;) plots the daily log price returns. Fat-tails displaying power-law behaviour with exponents in the range [2.8,3.2] are observed [5] (together with kurtosis values in the approximate range $[10,50]$ ). Finally Figure 101$)$ plots the autocorrelation functions of the price returns 

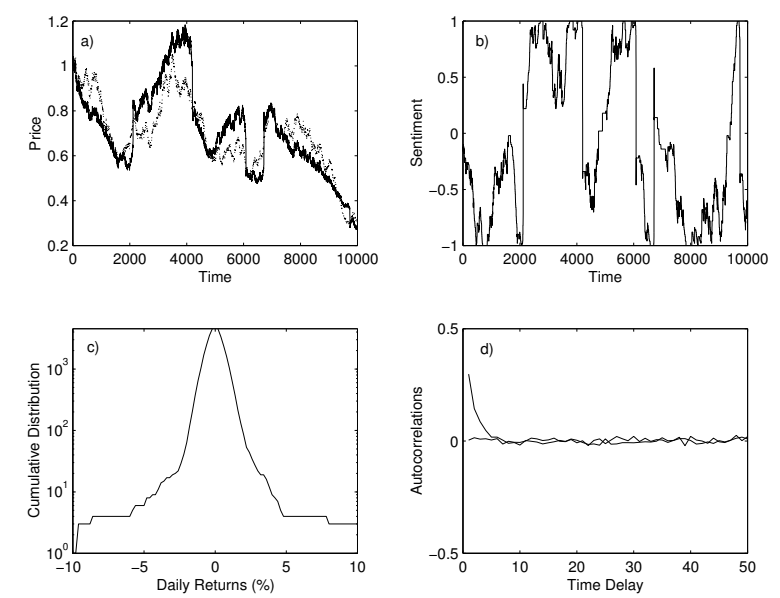

FIG. 1: Results of a simulation over 10000 timesteps. See the text for details.

and absolute price returns, a measure of volatility. The price returns show no evidence of linear correlations even with a lag of one day. The volatility correlations however die away after approximately 5 days or so. This lack of long-term volatility correlations or memory is the subject of the next section.

To recap, the introduction of herding does indeed generate fat-tails with decay rates that fit values extracted from actual market data. Further details, together with a 'computational experiment' that shows how to generate secondorder effects such as observed asymmetries in the price return data with respect to positive and negative price moves can be found in [5].

\section{SIMULATING CLUSTERED VOLATILITY}

Market models must be able to approximate the statistical properties of the market volatility which we define as the absolute $\log$-price return $\left|\log \frac{p(n+1)}{(p(n)}\right|$. However the causes of volatility clustering and long-memory are still poorly understood and there are several plausible mechanisms, all of which may play a significant role. There have been numerous studies investigating the relationship between volatility and other market variables, such as trading volume, but the question is still far from being resolved.

One possibility is that the clustering is due to non-stationarity and/or long-time correlations in the data stream. This is certainly plausible - geopolitical events and changes in economic conditions are rarely revealed by a single pulse of information entering the market, but rather unfold over a period of time. For the models of Section $\amalg$ and III these effects could be incorporated by replacing $\eta(n)$ with time series derived from fractional Brownian processes, stochastic volatility models, or GARCH-type processes (although one must be careful to ensure that no correlations are introduced into the returns themselves 13 ). However, certainly within the context of heterogeneous agent models (HAMs), these possibilities tend to be ignored, perhaps because it is more interesting to develop market 'black boxes' where all the non-Gaussian effects are generated internally. It is also possible to generate volatility clustering within HAMs via inductive learning and evolutionary strategies. To include such effects into our threshold models is certainly achievable (by choosing the inaction thresholds $H_{i}$ to reflect the agents' current strategy) but the resulting models are extremely complex and will not be considered here.

In the majority of HAMs that display clustered volatility, the underlying mechanism appears to be the ability of agents to switch between different 'fundamentalist' and 'chartist' strategies (for example, the Lux-Marchesi model 14]). Fundamentalist traders are betting that the price will quickly revert to some underlying rational price while the chartists believe that the recent price-trend will continue. Bubbles and anti-bubbles occur whenever the proportion of chartists exceeds some critical value. In our threshold models the agents are all of the same qualitative type so this switching between strategies cannot occur. However, the $M$ agents being explicitly simulated do not constitute the entire market since short-term noise traders are excluded. We now hypothesize that the number and activity-level of these traders is not constant in time but instead depends upon market conditions. The simplest scenario is that their effect upon the market is a function of overall sentiment. There is some evidence to support this correlation between volatility and (both bullish and bearish) sentiment from closed-end investment funds [15] (together with strong indications that the increases in volatility during times of extreme market sentiment were indeed due to noise traders rather than excess trading by fundamentalist investors). 

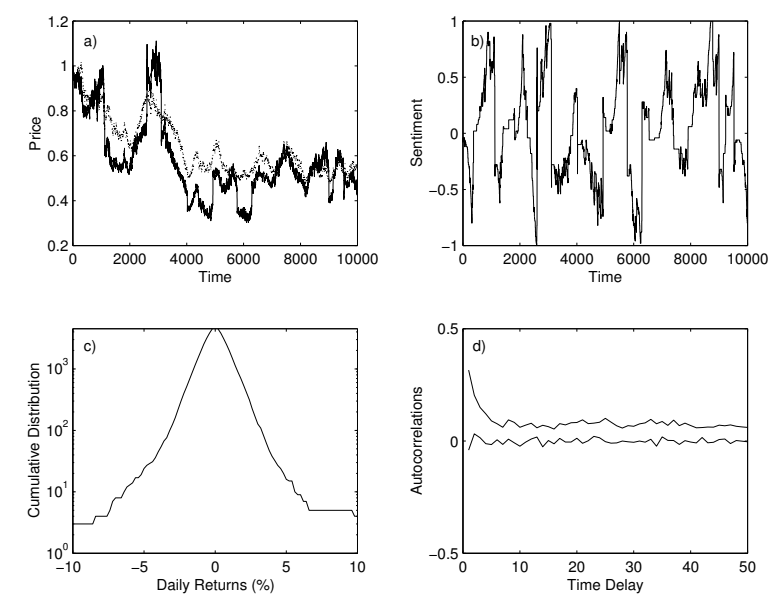

FIG. 2: The same data are plotted as in Figure 1 with but using the pricing formula (3).

Thus we replace the pricing formula (2) with

$$
p(n+1)=p(n) \exp ((\sqrt{h} \eta(n)-h / 2) f(\sigma)+\kappa \Delta \sigma(n))
$$

and assume a simple linear dependence of $f$ upon $|\sigma|$, i.e. $f(\sigma)=1+\alpha|\sigma|$ (setting $\alpha=0$ reverts to the model of Section III). A value of $\alpha=2$ (keeping all other parameters unchanged from Section III) does indeed add volatility clustering as can be seen in Figure 2. The rate of decay of the volatility autocorrelation function is an approximate power-law with exponent in the range $0.3-0.5$.

It should be noted that these threshold models are non-Markovian since the agents' tension levels are highly dependent upon the past behaviour of the system. This memory effect seems to be fundamental to the formation and collapse of the extended periods of mis-pricing that occur (and the corresponding fat-tails). However, the long-time volatility correlation introduced by (33) is not due to memory-effects. Rather, the price volatility due to external information now depends, via the function $f(\sigma)$ in (3), upon a slowly-changing system variable, namely the sentiment $\sigma$, and inherits its slow autocorrelation decay.

\section{PROFITABILITY OF TRADERS}

Finally we perform an interesting numerical experiment. Note that the agents' inaction thresholds change at every switching (to reflect updated future expectations) but their herding thresholds do not. This is because we consider the latter to be a measure of each agent's trading philosophy or personality and so more likely to remain constant over time. This raises the question of whether there is an observable difference in the relative performance between agents whose threshold values $C_{i}$ lie within the range [0.001,0.004] used in the simulations. Such a difference would suggest, within this modeling framework, the possibility of elementary, but effective, inductive learning strategies that simply consist of agents 'training' themselves to change their herding propensity.

To answer this question we keep track of the agents' profit or loss at each transaction during the simulation (note that the agents' financial performance does not affect their behaviour, although the reproduction of more realistic psychological pressures would probably include factors such as these). The agents are always assumed to hold \pm 1 units of the underlying asset and an inexhaustible cash supply to fund the transactions. The performance over the first 1000 timesteps is ignored to exclude transient effects caused by the externally imposed initial conditions.

The performance of the agents is displayed in Figure 3 where the overall profit/loss is plotted against that agent's herding threshold $C_{i}$. There is no significant correlation between profits and herding propensity. And of course if transaction costs are taken into account then agents with lower thresholds would actually perform relatively worse.

\section{CONCLUDING REMARKS}

The class of threshold HAMs studied here can incorporate enough psychology to describe realistic market behaviour. They are, however, difficult to analyse. But since all the coupling is global, a mean-field approach is possible. The 


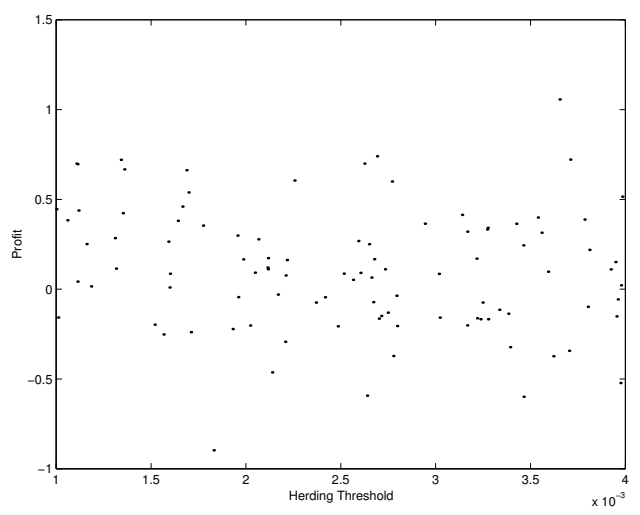

FIG. 3: The profit/loss of each agent plotted against their herding threshold. No significant correlation is observed.

resulting objects are stochastic difference equations coupled to deterministic ones; see [16] for an initial study of such a model, which, surprisingly, manages to reproduce some degree of volatility clustering without an explicit mechanism such as in (3). In future, we aim to use methods of discrete random dynamical systems [17] in order to elucidate, inter alia, the reasons for the appearance of power laws in the system.

[1] E. Fama, J. Finance 25, 383 (1970).

[2] R. Mantegna and H. Stanley, An Introduction to Econophysics (CUP, 2000).

[3] R. Cont, Quantitive Finance 1, 223 (2001).

[4] R. Cross, M. Grinfeld, H. Lamba, and T. Seaman, Phys. A 354, 463 (2005).

[5] H. Lamba and T. Seaman, preprint, Econophysics forum.

[6] B. LeBaron, in Post-Walrasian Economics, edited by D. Colander (CUP, New York, 2006).

[7] R. Cross, M. Grinfeld, H. Lamba, and A. Pittock, in Relaxation Oscillations and Hysteresis, edited by M. Mortell, R. O. Jr., A. Pokrovskii, and V. Sobolev (SIAM, 2005), pp. 61-72.

[8] B. Malkiel, Journal of Economic Perspectives 17, 59 (2003).

[9] A. Schleifer, Inefficient Markets, Clarendon Lectures in Economics (OUP, 2000).

[10] H. Simon, Quart. J. Econ. 69, 99 (1955).

[11] H. Simon, Models of Bounded Rationality (MIT Press, 1997).

[12] C. Chamley, Rational Herds (CUP, 2004).

[13] R. Baillie, T. Bollerslev, and H. Mikkelsen, J. Econometrics pp. 3-30 (1996).

[14] T. Lux and M. Marchesi, Int. J. Theor. Appl. Finance 3, 675 (2000).

[15] G. Brown, Financial Analysts Journal pp. 82-90 (1999).

[16] R. Cross, M. Grinfeld, and H. Lamba, preprint, submitted to J. de Physique.

[17] P. Diaconis and D. Freedman, SAM Review 41, 45 (1999). 